\title{
Amelioration of Mugwort on the Obesity and Dyslipidemia Induced by High-Fat Diet
}

\author{
Min-Ah Kim¹, Kye Won Park², Mi-Ja Kim, ${ }^{1, *}$ \\ ${ }^{1}$ Department of Food and Nutrition, College of Health Science, Kangwon National University, Samcheok, Gangwon 25949, Korea \\ ${ }^{2}$ Department of Food Science and Biotechnology, Sungkyunkwan University, Suwon 16419, Korea \\ *Corresponding author: mijakim@kangwon.ac.kr
}

Received September 18, 2019; Revised October 20, 2019; Accepted October 24, 2019

\begin{abstract}
Anti-obesity effects of Mugwort were evaluated in high fat diet-induced obesity mice models through determining physical changes and gene expression levels. High fat diet (HFD) models fed with 1\% or 3\% Mugwort had significantly lower body weight gain and food intake than HFD group $(p<0.05)$. Especially, body weight gain and food intake of 3\% Mugwort fed groups significantly decreased by $48.5 \%$ and $25.8 \%$, respectively, compared to HFD group. In addition, parameters in plasma including LDL levels, concentration of plasma triglyceride and total cholesterol as well as histological changes in the liver were reduced in Mugwort supplemented groups compared to the HFD. Addition of Mugwort decreased the gene expression levels of epididymal adipose tissue genes including ACC, GPDH, and PPAR $\gamma$. Therefore, Mugwort supplementation may participate in regulating plasma and hepatic steatosis as well as down-regulating adipogenic target gene expression.
\end{abstract}

Keywords: Mugwort, steatosis, fat accumulated genes, anti-obesity

Cite This Article: Min-Ah Kim, Kye Won Park, and Mi-Ja Kim, “Amelioration of Mugwort on the Obesity and Dyslipidemia Induced by High-Fat Diet.” Journal of Food and Nutrition Research, vol. 7, no. 10 (2019): 736-741. doi: 10.12691/jfnr-7-10-7.

\section{Introduction}

Obesity, a global health problem, is a health risk factor that causes insulin resistance, hypertension, dyslipidemia, cardiovascular disease, non-alcoholic fatty liver diseases, and cancer [1,2]. Recently, treatment strategies for obesity management are focusing on medicines that can suppress appetite, inhibit nutrient absorption, promote lipid oxidation, and inhibit lipid synthesis [3]. Lipid synthesis is mainly performed in adipose tissue. Adipogenesis causes multipotent mesenchymal stem cells to differentiate into mature adipocytes [4]. Homeostasis of lipid metabolism in adipose tissue is regulated by PPAR $\gamma$ and members of the C/EBP family as major transcription factors in adipogenesis $[4,5]$. Other transcription factors also play an essential role in the overall regulation of lipogenesis [6]. These transcription factors can influence the regulation of enzymes involved in lipid synthesis such as adipose specific fatty acid binding protein (aP2), cytosolic enzyme glycerol-3-phosphate dehydrogenase (GPDH), and acetyl CoA carboxylase (ACC) in downstream $[4,7,8]$. Although pharmacological treatments for obesity have been recently conducted, a few medicines are allowed for clinical use. Various complications can occur during short-term or long-term of medicine treatment to improve obesity. Therefore, it is necessary to find stable food materials for obesity management during a whole life-time. Many researches are concentrated on finding natural resources that can effectively control body weight without serious side effects [9-11].
Mugwort (artemisia lavandulaefolia) is a perennial herb that grows widely in mountains and fields of Korea [12]. It has been extensively used as a food ingredient. In addition, it has been used in traditional Korean medicine to prevent and treat various diseases, including allelopatic effects, gastrointestinal disorders, constipation, pain, asthma, and gynecological symptoms [13]. Furthermore, it is used as an anthelmintic and effective odor remover. Its essential oil ingredient is known to have anti-bacterial and anti-microbial action against many pathogenic bacteria and fungi in Chinese medicine [13,14,15]. Although there have been many studies on the pharmacological effect of Mugwort, its anti-obesity effect as food has not been reported yet. Therefore, the objective of present study was to examine the effect of Mugwort as food supplement on obesity and lipid profiles in high-fat induced obesity mice model.

\section{Materials and Methods}

\subsection{Animals and Diets}

Mugwort was bought at Kyungdong market (Seoul, Korea). Four-week old male C57BL/6 mice (Jung Ang Lab. Animal Inc. Seoul, Korea) were individually housed in cages and placed in a room with $12 \mathrm{~h}$ light-dark cycle and at $24^{\circ} \mathrm{C}$. After arrival, mice were fed commercial chow diet for one week to adapt. To induce obesity, mice were fed a high fat diet (HFD) (\% kcal; carbohydrate:protein:fat $=20: 20: 60)$ containing lard for 4 
weeks. Normal control group fed an AIN-76 based standard diet (STD) for 4 weeks. HFD fed mice were then randomly divided into three groups as follows: high fat diet (HFD), high fat diet with 1\% Mugwort (HFD+MW1\%), and high fat diet with 3\% Mugwort (HFD+MW3\%) groups. Body weight and food intake were measured every three days during the feeding period of 8 weeks. All mice were freely accessed to chow and water. The animal study was approved by the Sungkyunkwan University Institutional Animal Care and Use Committee (SKKU-IACUC, approval number: 2015-0037).

\subsection{Animal Treatment and Plasma Lipid Assay}

All mice were fasted for $15 \mathrm{~h}$ prior to being sacrificed. Animals were anesthetized using Zoletil. Blood samples were taken from the abdominal aorta and centrifuged to collect plasma samples for biochemical analysis. Liver and epididymal fat tissues were dissected. All samples were stored at $-70^{\circ} \mathrm{C}$ until analyzed. Plasma triglyceride (TG), total cholesterol (TC), high density lipoprotein (HDL) cholesterol, aspartate aminotransferase (AST), and alanine aminotransferase (ALT) concentrations were assayed using commercial kits (Sigma-Aldrich, St. Louis, MO, USA).

\subsection{Blood Glucose and Glucose Tolerance Test}

At the end of 12 weeks of experimental period, intraperitoneal glucose tolerance test (IPGTT) was executed in overnight fasted mice. Blood glucose was measured at basal state ( $0 \mathrm{~min})$ and at $15,30,60$, and 90 min after a glucose loading (2 g/kg, i.p.). Blood glucose concentrations were measured using a One touch Basic glucose measurement system (Life Scan Inc., Wayne, PA, USA) while taking blood from the tail vein. Mice were killed by decapitation immediately at $120 \mathrm{~min}$ after glucose loading.

\subsection{Hepatic and Epididymal Fat Tissues Histology}

Hepatic and epididymal fat tissues were immediately collected from the same location and fixed in $10 \%$ formalin solution for $24 \mathrm{~h}$. These hepatic and epididymal fat tissues were subsequently dehydrated with a series of ethanol solution ranging from 75 to $100 \%$ before they were embedded in paraffin wax. Cross-sections $(4 \mu \mathrm{m}$ in thickness) were cut and stained with hematoxylin and eosin followed by examination with a light microscope (Olympus Optical Co., Tokyo, Japan).

\subsection{Total RNA Extraction and Reverse Transcription Polymerase Chain Reaction (RT-PCR) Analysis}

Total RNA was obtained from epididymal fat tissue using TRI reagent (Sigma-Aldrich). According to the protocol, the reverse transcription (RT) was performed at $42{ }^{\circ} \mathrm{C}$ for $1 \mathrm{~h}$ followed by heating at $95{ }^{\circ} \mathrm{C}$ for $5 \mathrm{~min}$ to inactivate Avian Myeloblastosis Virus Reverse Transcriptase (AMV-RT) enzyme using commercial kit (Promega Co., Madison, WI , USA). Primer sequences for adipose specific fatty acid binding protein (aP2), peroxisome proliferation-activated receptor $\gamma$ (PPAR $\gamma$ ), glycerol-3-phosphate dehydrogenase (GPDH), and acetyl CoA carboxylase (ACC) and their amplification conditions using RT-PCR were provided as supplementary data. PCR products were loaded onto $1.5 \%$ agarose gel stained with ethidium bromide. Relative level of RT-PCR product was measured with Frog gel image analysis system (Spot Densitometry Program, Core Bio, Seoul, Korea) under UV light.

\subsection{Statistical Analysis}

Data are presented as mean \pm SEM and analyzed by one-way analysis of variance (ANOVA) followed by Duncan's multiple range test using SPSS version 23 (SPSS Inc., Chicago, IL, USA). Statistical significance was determined at $p<0.05$.

\section{Results and Discussion}

\subsection{Effect of Mugwort on Body Weight and Food Intake}

Body weight gain and food intake of animals fed with Mugwort are shown in Table 1. HFD group increased body weight and food intake significantly for 8 weeks $(p<0.05)$. Body weight gain of the HFD group increased by $50.9 \%$ compared to that of the STD group whereas those of the HFD group supplemented with $1 \%$ or $3 \%$ Mugwort significantly decreased by $33.7 \%$ or $48.5 \%$, respectively $(p<0.05)$ compared to that of the HFD group without such supplementation. Food intake of the HFD group supplemented with $1 \%$ or $3 \%$ Mugwort was significantly decreased compared to that of the HFD group $(p<0.05)$. Food intake of the HFD group supplemented with $3 \%$ Mugwort was similar to that of the STD group (Table 1).

Table 1. Effect of Mugwort on body weight and food intake in high fat diet induced obese mouse

\begin{tabular}{lcccc}
\hline & STD & HFD & HFD+MW1\% & HFD+MW3\% \\
\hline $\begin{array}{l}\text { Initial body } \\
\text { weight (g) }\end{array}$ & $25.6 \pm 0.5^{\mathrm{a}}$ & $30.9 \pm 0.9^{\mathrm{b}}$ & $30.7 \pm 0.8^{\mathrm{b}}$ & $30.6 \pm 0.6^{\mathrm{b}}$ \\
$\begin{array}{l}\text { Final body } \\
\text { weight (g) }\end{array}$ & $36.4 \pm 0.4^{\mathrm{d}}$ & $47.2 \pm 0.4^{\mathrm{a}}$ & $41.6 \pm 1.0^{\mathrm{b}}$ & $38.9 \pm 0.8^{\mathrm{c}}$ \\
$\begin{array}{l}\text { Body weight } \\
\text { gain(g) }\end{array}$ & $10.8 \pm 0.4^{\mathrm{b}}$ & $16.3 \pm 1.4^{\mathrm{a}}$ & $10.8 \pm 1.0^{\mathrm{b}}$ & $8.4 \pm 0.8^{\mathrm{c}}$ \\
$\begin{array}{l}\text { Food } \\
\text { intake(g/day) }\end{array}$ & $4.00 \pm 0.04^{\mathrm{c}}$ & $5.43 \pm 0.18^{\mathrm{a}}$ & $4.43 \pm 0.09^{\mathrm{b}}$ & $4.03 \pm 0.21^{\mathrm{c}}$ \\
\hline
\end{tabular}

Data are mean $\pm \operatorname{SEM}(n=10)$. STD, standard diet fed group; HFD, high fat diet fed group, HFD+MW1\%, high fat diet supplement with $1 \%$ Mugwort, HFD+MW3\%, high fat diet supplement with 3\% Mugwort. Different letters denote means significant differences among groups $(p<0.05)$.

Effects of Mugwort on epididymal fat tissues in high fat diet induced obese mouse are shown in Figure 1. The weight reduction effects of Mugwort were clearly observed with histologic changes of epididymal 
fat tissue. Sizes of adipocytes stained with epididymal WAT were remarkably larger in the HFD group than those in Mugwort supplement groups (Figure 1). Mugwort may decrease adipocyte size by reducing lipid accumulation in fat tissue, resulting in decreased body weight.
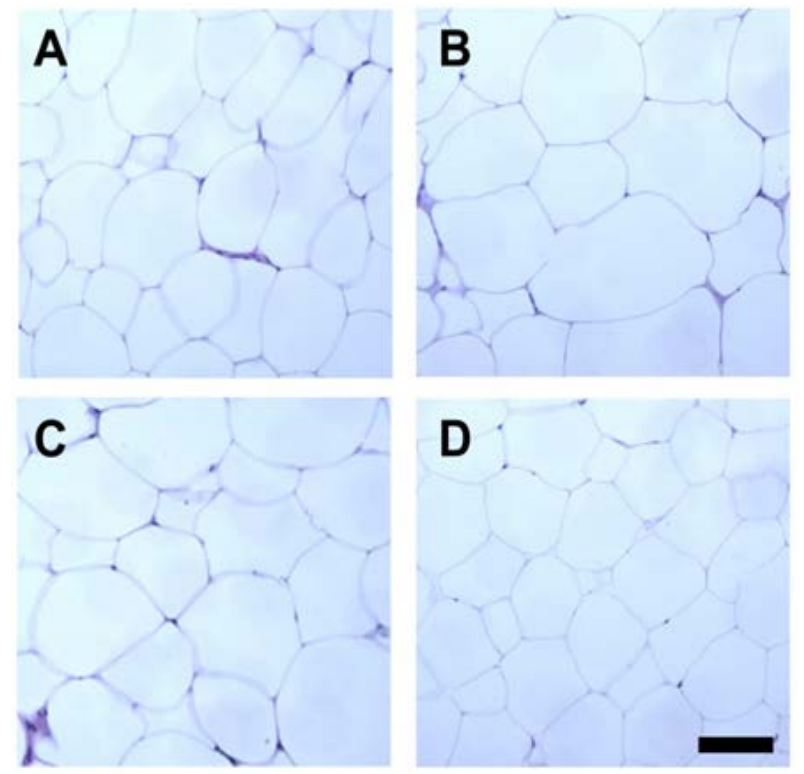

Figure 1. Mugwort supplementation on epididymal fat tissues in high fat diet induced obese mouse. (A STD, standard diet fed group; B HFD, high fat diet fed group, C HFD+MW1\%, high fat diet supplement with 1\% Mugwort, D HFD+MW3\%, high fat diet supplement with 3\% Mugwort. Scale bar represents $50 \mu \mathrm{m}$.)

Mugwort is known to contain a variety of minerals as well as phenolics, alkaloids, and bioactive compounds such as vitamins A, B1, B2, and C [16]. These compounds in Mugwort might affect weight reduction of animals with obesity induced by high fat diet.

\subsection{Effect of Mugwort on Plasma Lipid Profiles}

Changes of plasma TG, total cholesterol, LDLcholesterol, and HDL-cholesterol levels of experimental groups are summarized in Table 2. For the HFD group, the plasma levels of TG, total cholesterol, and LDL-cholesterol levels significantly increased by 35.7 , 57.9, and $125.7 \%$, respectively $(p<0.05)$, whereas plasma HDL cholesterol levels were significantly $(p<0.05)$ reduced by $15.2 \%$ compared to those in the STD group. HFD fed with 1\% Mugwort had significantly reduced plasma levels of TG, total cholesterol, and LDL cholesterol by 31.3, 31.6, and 50.0\%, respectively $(p<0.05)$ while HDL cholesterol levels significantly increased by $31.0 \% \quad(p<0.05)$ compared to those in the HFD group. In the HFD supplemented with 3\% Mugwort, the plasma concentrations of TG, total cholesterol, and LDL-cholesterol were lower by 32.8, 40.2 and $65.7 \%$, respectively, while HDL-cholesterol was higher by $39.4 \%$ than those of HFD group. Mugwort supplementation is effective in lowering LDL-cholesterol among total lipids elevated by high fat diet and ameliorated the phenomena of dyslipidemia found in HFD
Table 2. Effect of Mugwort on lipid profiles in blood

\begin{tabular}{rcccc}
\hline & STD & HFD & HFD+MW1\% & HFD+MW3\% \\
\hline TG & $135.4 \pm 3.8^{\mathrm{b}}$ & $183.7 \pm 1.6^{\mathrm{a}}$ & $126.2 \pm 2.5^{\mathrm{bc}}$ & $123.4 \pm 2.0^{\mathrm{c}}$ \\
TC & $126.2 \pm 4.2^{\mathrm{bc}}$ & $199.3 \pm 2.5^{\mathrm{a}}$ & $136.3 \pm 2.6^{\mathrm{b}}$ & $119.1 \pm 3.3^{\mathrm{c}}$ \\
LDL & $55.7 \pm 4.2^{\mathrm{b}}$ & $125.7 \pm 2.5^{\mathrm{a}}$ & $62.8 \pm 2.6^{\mathrm{b}}$ & $43.1 \pm 3.3^{\mathrm{c}}$ \\
HDL & $43.4 \pm 1.9^{\mathrm{b}}$ & $36.8 \pm 1.0^{\mathrm{c}}$ & $48.2 \pm 1.5^{\mathrm{ab}}$ & $51.3 \pm 0.9^{\mathrm{a}}$ \\
\hline
\end{tabular}

Data are mean $\pm \operatorname{SEM}(n=10)$. STD, standard diet fed group; HFD, high fat diet fed group, HFD+MW1\%, high fat diet supplement with $1 \%$ Mugwort, HFD+MW3\%, high fat diet supplement with 3\% Mugwort. Different letters denote means significant differences among groups $(p<0.05)$.

Jang et al. [17] have studied the effect of artemisia capillaris on hyperlipemia and cardiovascular diseases and reported that total cholesterol, triglyceride, and malondialdehyde decreased by supplemented with artemisia capillaris water extract. Artemisia capillaris has been reported to be involved in the down-regulation of microRNA-122 induced fatty acid synthase in vitro and in vitro systems, thereby reducing plasma levels of triglyceride, total cholesterol, and LDL-cholesterol [18]. In addition, artemisia capillaris Thumberg essential oil components possessing coumarin, $\beta$-citronellol, 1,8-cineole, camphor, linalool, $\alpha$-pinene, $\beta$-pinene, thymol, and myrcene could be used to treat jaundice and eczema [19]. Because these components can successfully stimulate secretion of bile, they have been used to treat inflammation and hepatitis [20]. Taken together, these results suggest that Mugwort plays an important role in blood lipid metabolism dysfunction by high fat diet.

\subsection{Effect of Mugwort on Plasma Glucose and Glucose Tolerance}

Changes of glucose concentration (A) and tolerance (B) in high fat diet induced obese mouse fed with Mugwort are shown in Figure 2. Blood glucose levels in the HFD group were significantly higher than those in the STD group $(p<0.05)$ (Figure 2A). The glucose tolerance of the HFD group was observed more those of other groups according to glucose loading at $0,30,60,90$, and 120 min (Figure 2B). The pattern of glucose tolerance was similar to that of plasma glucose concentration in all experimental groups after glucose loading. However, blood glucose concentration in the HFD group failed to return to normal levels at $30 \mathrm{~min}$ after glucose loading. Mugwort supplement at 1 and 3\% significantly reduced fasting blood glucose and significantly improved glucose tolerance compared to the HFD group $(p<0.05)$. Therefore, diet containing Mugwort could decrease significantly HFD-induced hyperglycemia in a dose-dependent manner. Although hyperlipidemia depends on the type of diet, hyperglycemia usually develops within 4 weeks of a high-fat diet [21]. Fasting blood glucose elevation is usually accompanied by an increase in fasting insulin levels, therefore showing insulin resistance. It is known that fasting insulin elevation stimulates triglyceride synthesis in adipose tissue and exacerbates obesity [22]. Abnormal accumulation of fat occurs in insulin-sensitive tissues other than adipose tissues. Such accumulation is considered a major cause of insulin resistance [23]. A strong correlation is found between intracellular 
triglyceride accumulation and insulin resistance [24,25]. In this study, high fat diets resulted in hyperglycemia and impaired glucose tolerance while Mugwort supplementation ameliorated these results in obese mice models.

\section{A)}

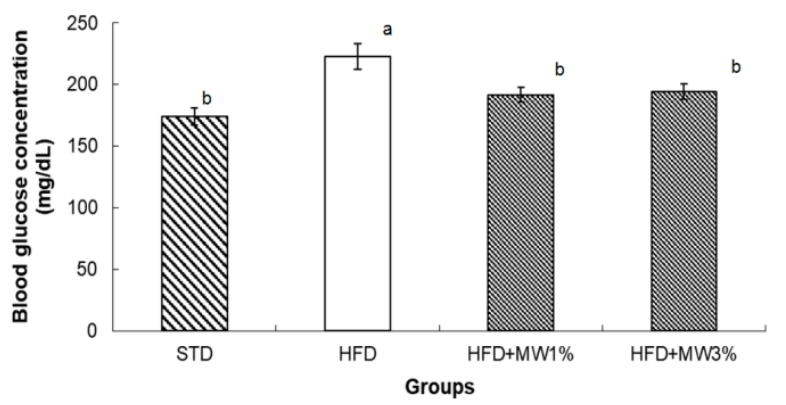

B)

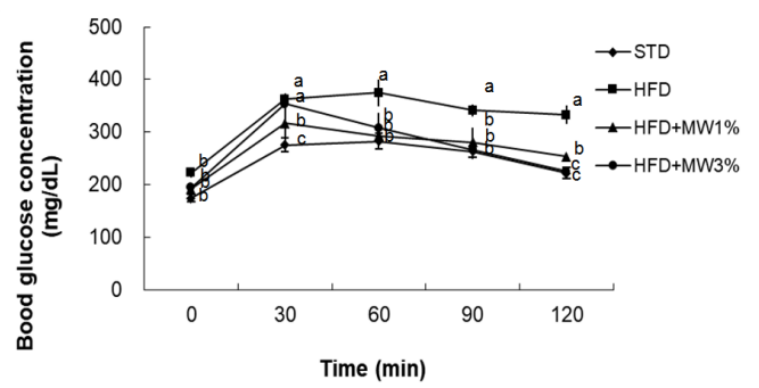

Figure 2. Changes of glucose concentration (A) and tolerance (B) in high fat diet induced obese mouse fed with Mugwort. (STD, standard diet fed group; HFD, high fat diet fed group, HFD+MW1\%, high fat diet supplement with $1 \%$ Mugwort, HFD+MW3\%, high fat diet supplement with 3\% Mugwort. Different letters denote means significant differences among groups $(p<0.05)$.)

\subsection{Effect of Mugwort on Plasma Levels of AST and ALT as Well as Histological Changes of the Liver}

Effects of Mugwort supplement on blood AST and ALT (A) and hepatic tissue morphology (B) in high fat diet induced obese mouse are shown in Figure 3. Plasma AST and ALT concentrations significantly increased in HFD group whereas Mugwort supplemented group normalized these parameters (Figure 3A). Plasma levels of AST and ALT in HFD group increased significantly by 62.2 and $134.7 \%$, respectively, compared to those in the STD group $(p<0.05)$. However, supplementation with $1 \%$ Mugwort in the HFD group significantly reduced AST and ALT levels by 28.4 and 45.1\%, respectively, compared to the HFD group $(p<0.05)$ while AST and ALT levels of groups with 3\% Mugwort lowered by 31.8 and $53.9 \%$ than the HFD group, respectively. Furthermore, the HFD group showed accumulation of hepatic lipid droplets and severe steatosis compared to the STD group possessing normal hepatic histology (Figure 3B-B). However, Mugwort supplementation remarkably diminished the extent of steatosis. This result suggests that Mugwort may regulate lipid mobilization and storage in adipocytes of major tissues.

Generally, a high fat diet can increase fatty acids synthesis in the liver and the transfer of free fatty acids to the liver [26], thereby reducing $\beta$-oxidation of free fatty acids. This will result in fat accumulation in the liver [27]. Therefore, fatty liver is caused by many factors, including excessive blood levels of free fatty acids, more fatty acid synthesis, exaggeration with esters, reduction of fatty acid oxidation rate, and decrease of apolipoprotein synthesis alone or in combination.

Kim et al. [28] reported that extracts of artemisia capillaris Thumberg could reduce the blood levels of AST, ALT, and liver microsomal peroxides in a CCl4-induced liver injury model. As shown in Figure 3B-B, liver tissues in the HFD group showed macrovesicular steatosis. However, Mugwort supplementation diminished the extent of steatosis (Figure. 3B-C and -D).

A)

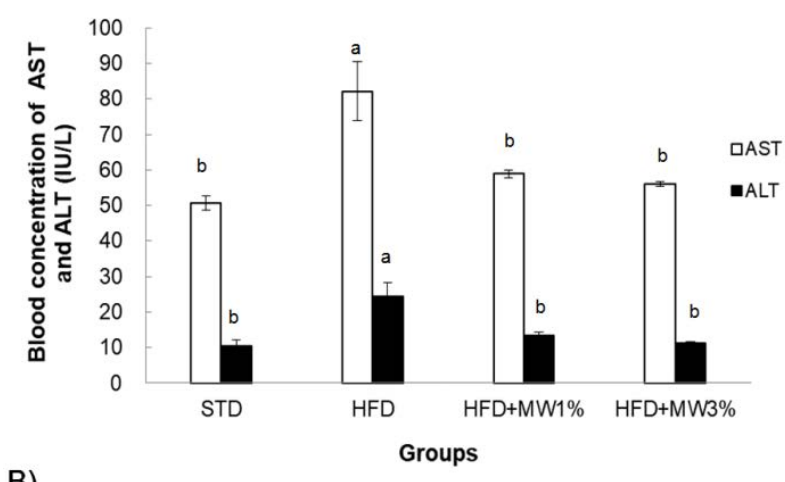

B)
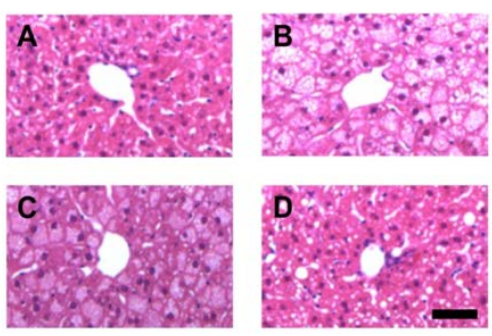

Figure 3. Effects of Mugwort supplement on blood AST and ALT (A) and hepatic tissue morphology (B) in high fat diet induced obese mouse. (A) Blood concentrations of AST and ALT in high fat diet induced obesity model. B) Hepatic tissue morphology in diet induced obesity model. A: STD, standard diet fed group; B: HFD, high fat diet fed group, C: HFD+MW1\%, high fat diet supplement with $1 \%$ Mugwort, D: HFD+MW3\%, high fat diet supplement with 3\% Mugwort. Different letters denote means significant differences among groups $(p<0.05)$. Scale bar represents $50 \mu \mathrm{m}$.)

\subsection{Effect of Mugwort on Fat Accumulated Gene Expression in Epididymal Fat}

Fat accumulating genes expression (A) and optical densities (B) in epididymal fat tissues of high fat diet induced obese mouse fed with Mugwort are shown in Figure 4. RT-PCR analysis was performed to determine whether Mugwort affected the expression of adipogenic genes in epididymal fat tissues (Figure 4). HFD had higher mRNA expression levels of aP2, PPAR $\gamma, \mathrm{GPDH}$, and ACC than STD group. However, mRNA expression levels of aP2 and PPAR $\gamma$ in the group supplemented with $1 \%$ Mugwort significantly $(p<0.05)$ decreased by 42.6 and $25.0 \%$, respectively, and by 33.2 and $95.1 \%$, respectively, in the 3\% Mugwort group compared to those in the HFD group (Figure 4B). 
A)

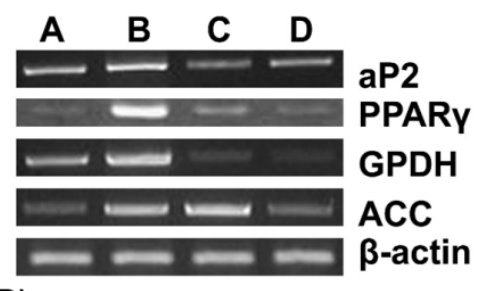

B)

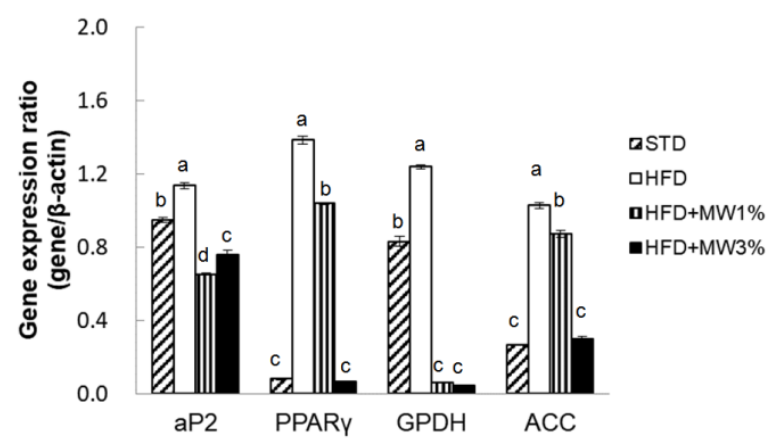

Figure 4. Fat accumulating genes expression (A) and optical densities (B) in epididymal fat tissues of high fat diet induced obese mouse fed with Mugwort. (A) A: STD, standard diet fed group; B: HFD, high fat diet fed group, C: HFD+MW1\%, high fat diet supplement with 1\% Mugwort, D: HFD+MW3\%, high fat diet supplement with 3\% Mugwort. B) Optical densities of genes expression related to fat accumulation in epididymal fat tissues of high fat diet induced obesity model.

In addition, HFD with $1 \%$ Mugwort had lower expression levels of GPDH and ACC mRNAs by 95.2 and $15.2 \%$, respectively. For the groups of $3 \%$ Mugwort, expression levels of GPDH and ACC mRNAs decreased by 96.2 and $70.7 \%$, respectively compared to the HFD group.

Supplementation of 3\% Mugwort was more effective than that $1 \%$ Mugwort in inhibiting expression of lipogenic genes such as PPAR $\gamma$, GPDH, and ACC. Fatty acid is the most essential substrate for biosynthesis of TG and PPAR $\gamma$ is well-known as an important transcription factor [29] mainly expressed in adipose tissue that plays a vital role in adipocyte differentiation, lipid storage, and glucose homeostasis [30]. PPAR $\gamma$ controls gene expression of enzymes involved in adipose tissue fat production, such as fatty acid FAS, aP2, ACC, and lipoprotein lipase (LPL) [31]. aP2 is a fatty acid transport protein that is mainly expressed in adipocytes and macrophages [32]. ACC [33] and GPDH [34] are also important enzymes involved in fat accumulation. GPDH is an enzyme that links glucose to lipid metabolism by facilitating the synthesis of glycerol-3-phosphate. Glycerol-3-phosphate produced by this reaction is a constituent of triglyceride in fat tissue [34]. RT-PCR results showed that the most effective dosage of Mugwort for adipogenesis inhibition was at 3\% based on results of expression levels of PPAR $\gamma$, GPDH, and ACC mRNA.

\section{Conclusion}

Mugwort supplementation has a beneficial effect by $48.5 \%$ reducing body weight gain and adipocyte size as well as lipid levels in plasma in HFD-induced obese model. In addition, Mugwort uptake was reduced by over
50\% LDL-cholesterol and improved glucose tolerance. These results suggest that Mugwort in HFD-induced obese mice may regulate lipid metabolism through inhibition of lipogenesis by down-regulating adipogenic transcription factor and other specific target genes. This study highlights the potential importance of Mugwort as an anti-obesity ingredient for the prevention of dyslipidemia.

\section{References}

[1] Funahashi, T. and Matsuzawa, Y., "Metabolic syndrome: Clinical concept and molecular basis”, Annals of Medicine, 39, 482-494, 2007.

[2] Lautt, W.W., “A new paradigm for diabetes and obesity: The hepatic insulin sensitizing substance (HISS) hypothesis”, Journal of Pharmacological Sciences, 95, 9-17, 2004.

[3] Hofbauer, K.G., Nicholson, J.R. and Boss, O., "The obesity epidemic: Current and future pharmacological treatments”, Annual Review of Pharmacology and Toxicology, 47, 565-592, 2007.

[4] Tontonoz, P., Hu, E. and Spiegelman, B.M., "Stimulation of adipogenesis in fibroblasts by PPAR $\gamma 2$, a lipid-activated transcription factor”, Cell, 79, 1147-1156, 1994.

[5] Zhao, X.Y., Chen, X.Y., Zhang, Z.J., Kang, Y., Liao, W.M., Yu, W.H. and Xiang, A.P., "Expression patterns of transcription factor PPAR $\gamma$ and C/EBP family members during in vitro adipogenesis of human bone marrow mesenchymal stem cells”, Cell Biology International, 39(4), 457-465, 2015.

[6] Wu, Z., Puigserver, P. and Spiegelman, B.M., "Transcriptional activation of adipogenesis”, Current Opinion in Cell Biology, 11, 689-694, 1999.

[7] Kim, M.J., Jeon, J. and Lee, J.S., "Fucoidan prevents high-fat dietinduced obesity in animals by suppression of fat accumulation", Phytotheraphy Research, 28, 137-143, 2014.

[8] Swierczynski, J., Zabrocka, L., Goyke, E., Raczynska, S., Adamonis, W. and Sledzinski, Z., "Enhanced glycerol 3-phosphate dehydrogenase activity in adipose tissue of obese humans”, Molecular and Cellular Biochemistry, 254, 55-59, 2003.

[9] Andersen, C., Rayalam, S., Della-Fera, M.A. and Baile, C.A., "Phytochemicals and adipogenesis", Biofactors, 36, 415-422, 2010.

[10] Mayer, M.A., Höcht, C., Puyó, A. and Taira, C.A., "Recent advances in obesity pharmacotherapy", Current Clinical Pharmacology, 4, 53-61, 2009.

[11] Wang, S., Moustaid-Moussa, N., Chen, L., Mo, H., Shastri, A., Su, R., Bapat, P., Kwun, I. and Shen, C.L., "Novel insights of dietary polyphenols and obesity”, The Journal of Nutritional Biochemistry, 25, 1-18, 2014.

[12] Bora, K.S. and Sharma, A., "The genus artemisia: A comprehensive review”, Pharmaceutical Biology, 49(1), 101-109, 2011.

[13] Abad, M.J., Bedoya, L.M., Apaza, L., Bermejo, P., “The artemisia L. Genus: A review of bioactive essential oils”, Molecules, 17(3), 2542-2566, 2012.

[14] Wink, M., "Plant breeding, importance of plant secondary metabolites for protection against pathogens and herbivores", Theoretical and Applied Genetics, 75, 225-233, 1988.

[15] Yun, K.W., Kil, B.S. and Han, D.M., "Phytotoxic and antimicrobial activity of volatile constituents of artemisia princeps var. orientalis”, Journal of Chemical Ecology, 19, 2757-2766, 1993.

[16] Hwang, K.E., Choi, Y.S., Choi, J.H., Kim, H.Y., Lee, M.A., Kim, H.W., Chung, H.K. and Kim, C.J., "The antioxidative properties of Ganghwayak ssuk (artemisia princeps pamp.) extracts added to refrigerated raw chicken nugget batter against lipid oxidation”, Korean Journal for Food Science of Animal Resources, 31, 166-175, 2011.

[17] Jang, W.S., Kim, Y.S. and Seol, I.C., Antioxidant and lipidlowering effects of artemisia capillaris on a rat model of hyperlipidemia. The Journal of Korean Oriental Medicine, 33(2), 11-24, 2012.

[18] Liu, L., Zhao, J., Li,Y., Wan, Y., Lin, J., Shen, A., Xu, W., Li, H., Zhang, Y., Xu, J., Peng, J. and Hong, Z., “Artemisia capillaris formula inhibits hepatic steatosis via an miR - 122 - induced 
decrease in fatty acid synthase expression in vivo and in vitro. Molecular Medicine Reports, 13(6), 4751-4758, 2016.

[19] Badoni, R., Semwal, D.K. and Rawat, U., "Composition variation in essential oils of artemisia nilagirica and artemisia capillaris, growing in India. Journal of Applied and Natural Science, 2(1), 30-33, 2010.

[20] Gaoa, Q., Zhaoa, X., Yin, L., Zhang, Y., Wang, B., Wu, X., Zhang, $\mathrm{X} ., \mathrm{Fu}, \mathrm{X}$. and Sun, W., "The essential oil of artemisia capillaris protects against CCl4-induced liver injury in vivo", Revista Brasileira de Farmacognosia, 26, 369-374, 2016.

[21] Sato, A., Kawano, H., Notsu, T., Ohta, M., Nakakuki, M., Mizuguchi, K., Itoh, M., Suganami, T. and Ogawa, Y., “Antiobesity effect of eicosapentaenoic acid in high-fat/high-sucrose diet-induced obesity: Importance of hepatic lipogenesis”, Diabetes, 59, 2495-2504, 2010.

[22] Rosen, E.D. and Spiegelman, B.M., “Adipocytes as regulators of energy balance and glucose homeostasis”, Nature, 444, 847-853, 2006.

[23] Shulman, G.I., "Cellular mechanisms of insulin resistance", Journal of Clinical Investigation, 106, 171-176, 2000.

[24] Guilherme, A., Virbasius, J.V., Puri, V. and Czech, M.P., "Adipocyte dysfunctions linking obesity to insulin resistance and type 2 diabetes”, Nature Reviews Molecular Cell Biology, 9(5), 367-377, 2008.

[25] Perseghin, G., Scifo, P., De Cobelli, F., Pagliato, E., Battezzati, A., Arcelloni, C., Vanzulli, A., Testolin, G., Pozza, G., Del Maschio, A. and Luzi, L., "Intramyocellular triglyceride content is a determinant of in vivo insulin resistance in humans: A $1 \mathrm{H}-13 \mathrm{C}$ nuclear magnetic resonance spectroscopy assessment in offspring of type 2 diabetic parents”, Diabetes, 48, 1600 -1606, 1999.

[26] Gauthier, M.S., Couturier, K., Latour, J.G. and Lavoie, J.M., "Concurrent exercise prevents high-fat-diet-induced macrovesicular hepatic steatosis”, Journal of Applied Physiology, 94, 2127-2134, 2003.

[27] Staiger, H. and Häring, H.U., “Adipocytokines: Fat-derived humoral mediators of metabolic homeostasis”, Experimental and Clinical Endocrine and Diabetes, 113, 67-79, 2005.

[28] Kim, D.W., Cho, H.I., Kim, K.M., Kim, S.J., Choi, J.S., Kim, Y.S. and Lee, S.M., "Isorhamnetin-3-O-galactoside protects against CCl4-Induced hepatic injury in mice”, Biomolecules and Therapeutics, 20(4), 406-412, 2012.

[29] Rosen, E.D., Walkey, C.J., Puigserver, P. and Spiegelman, B.M., "Transcriptional regulation of adipogenesis", Genes and Development, 14, 1293-1307, 2000.

[30] Schoonjans, K., Staels, B. and Auwerx, J., “The peroxisome proliferator activated receptors (PPARS) and their effects on lipid metabolism and adipocyte differentiation”, Biochimica et Biophysica Acta, 1302, 93-109, 1996.

[31] Kliewer, S.A. and Willson, T.M., "The nuclear receptor PPAR $\gamma$ bigger than fat", Current Opinion in Genetics and Development, 8, 576-581, 1998.

[32] Furuhashi, M., Fucho, R., Görgün, C.Z., Tuncman, G., Cao, H. and Hotamisligil, G.S., "Adipocyte/macrophage fatty acid-binding proteins contribute to metabolic deterioration through actions in both macrophages and adipocytes in mice”, Journal of Clinical Investigation, 118(7), 2640-2650, 2008.

[33] Tong, L., “Acetyl-coenzyme A carboxylase: Crucial metabolic enzyme and attractive target for drug discovery”, Cellular and Molecular Life Sciences, 62, 1784-1803, 2005.

[34] Moustaïd, N., Jones, B.H. and Taylor, J.W., "Insulin increases lipogenic enzyme activity in human adipocytes in primary culture”, Journal of Nutrition, 126, 865-870, 1996.

(C) The Author(s) 2019. This article is an open access article distributed under the terms and conditions of the Creative Commons Attribution (CC BY) license (http://creativecommons.org/licenses/by/4.0/). 